\title{
An Equivalent Model for Modal Analysis of Engine Mounting System
}

\author{
Shuxun Chen ${ }^{1, a}$, Qifeng Wei ${ }^{1, b}$, Jincheng Huang ${ }^{1, c}$, \\ Guobao Yin ${ }^{1, \mathrm{~d}}$ and Zhiqiang $\mathrm{Li}^{1, \mathrm{e}}$ \\ ${ }^{1}$ Guangxi University, Nanning, China \\ a chenshx@gxu.edu.cn, ${ }^{\mathrm{b}}$ weiqifeng21@126.com, ${ }^{\mathrm{c}}$ hujch@126.com, \\ dellor@163.com, e ynkmlzq2008@yahoo.com.cn
}

Keywords: Mounting system; Equivalent model; Modal analysis; Decoupling degree; ANSYS

\begin{abstract}
An equivalent analysis model for engine powertrain mounting system is built with the FEA software ANSYS, in which the engine powertrain is equivalent to a composite body of one inertial body and one elastic body, every mounting component is simplified as one spring along its three principal elastic axes and the powertrain mass and rotational inertia are accurately embodied. With this equivalent model, vibration modes and energy decoupling degrees can be calculated. The result of an example in ANSYS shows that this equivalent modeling method is validity and feasibility for engine powertrain mounting systems.
\end{abstract}

\section{Introduction}

An engine powertrain mounting system refers to a system comprising of an engine powertrain and elastic connecting components between the engine powertrain and the vehicle frame or vehicle body, shown in Fig.1, where the red circled parts are mounting components. A mounting system shall have good effect of vibration isolation: it is to prevent the engine from transferring vibration forces to the vehicle frame, and to prevent the vibrations due to the excitation of uneven road surface from being passed to the engine. Meanwhile, it should be able to limit effectively the engine's maximum displacement to avoid collision and interference with adjacent parts, and thus to guarantee the normal running of the engine. Fig. 2 shows a schematic diagram of equivalent mode of an engine powertrain mounting system, where the circled parts are the simplified mounting components.

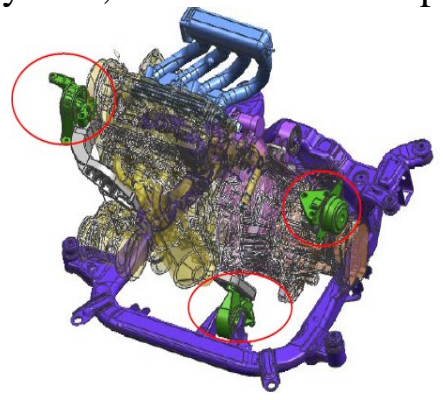

Fig.1 An engine mounting system

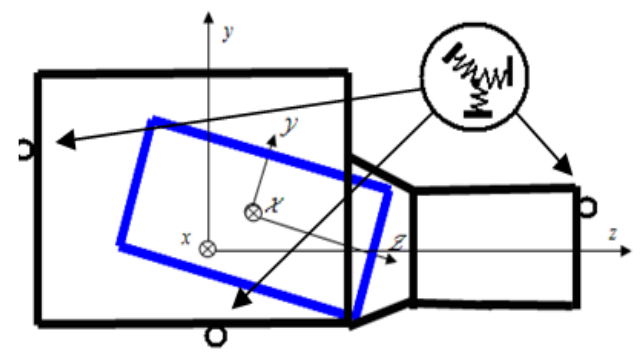

Fig.2 equivalent mode

The purpose of analysis and optimization for an engine mounting system [1-3] is to find the optimum design parameters such as angle of each principal elastic axis of every mounting component, location of elastic center and stiffness in various directions of each mounting component, so as to maximize the vibration isolation effect. At present, optimization methods for mounting systems [4-7] are mostly to treat the engine powertrain as a six-degree-of-freedom rigid body described by mass center, mass, moments of inertia, and products of inertia, mounting components as undamped springs, and the vehicle frame as a rigid foundation, and to establish an undamped free vibration equation:

$\left(\boldsymbol{K}-\omega^{2} \boldsymbol{M}\right) \varphi=0$

where, the mass matrix $\boldsymbol{M}$ is composed of the mass, the moments of inertia and the products of inertia of the engine powertrain; the stiffness matrix $\boldsymbol{K}$ depends on the location, the direction of elastic axis and the stiffness in various directions of mounting components; $\omega$ is the resonant angular 
frequency and $\varphi$ is the corresponding mode shape vector. By solving the generalized eigenvalues of Eq. (1), the six-natural frequencies and corresponding mode shapes can be obtained. Then, through the following equation, the energy decoupling degrees in various directions of each mode shape can be solved,

$$
T_{k}^{i}=\frac{\sum_{l=1}^{6}\left(\varphi_{i}\right)_{l}\left(\varphi_{i}\right)_{k} m_{k l}}{\sum_{l=1}^{6} \sum_{k=1}^{6}\left(\varphi_{i}\right)_{l}\left(\varphi_{i}\right)_{k} m_{k l}} \times 100 \%
$$

where $\left(\varphi_{i}\right)_{k}$ is the $k^{\text {th }}$ direction displacement in the $i^{\text {th }}$ mode shape, $m_{k l}$ is the element in the $k^{\text {th }}$ row and the $l^{\text {th }}$ column of mass matrix $\boldsymbol{M}$. Employing optimization algorithm[4-7] to maximize the decoupling degrees in various directions, and making the resonant frequencies to fall within an expected range, making the structural resonance frequencies to avoid the exciting force frequencies as far as possible, so as to indirectly improve vibration isolation effect of the mounting system.

This paper introduces an equivalent model of engine mounting system in ANSYS software, which is useful for resonant frequency, decoupling degree, dynamic response analysis and optimization of mounting systems.

\section{Construction of the equivalent analysis model}

In the equivalent analysis model, engine powertrain mounting system is equivalent to a composite body of one inertial body and one simplified elastic body, and every mounting component is simplified as one spring along its principal elastic axes. The inertial body is a cuboid of which the geometric center coincided with that of a real engine powertrain (Fig.4), its directions of three-dimensional sides are consistent with those of principal axes of inertia for the engine powertrain, and its mass and rotational inertia are strictly equal to their actual values. The inertial body reflects the mass distribution characteristic of an actual engine powertrain; Besides, The simplified elastic body is a quasi-geometric model of an engine powertrain, and accurate geometric modeling of the engine powertrain is not necessary, since the simplified elastic body is only used for connection of the mounting components and for reflection of the action points of engine exciting forces when refer to dynamic response analysis. The density of this elastic body is taken as zero, as the mass characteristic of the engine powertrain has been reflected in the inertial body. The elastic modulus of this elastic body can be taken as that of the engine material. The procedures for constructing an equivalent body of engine powertrain in ANSYS are as follows:

(1) Construct a matrix made from the engine powertrain parameters including moments of inertia and products of inertia, calculate the three principal moments of inertia $\left(I_{X X}, I_{Y Y}\right.$, and $\left.I_{Z Z}\right)$ and the direction cosine matrix $A$ included angles between the principal-axis-of-inertia coordinate system $O X Y Z$ and the vehicle-body coordinate system $O X Y Z$. The vehicle body coordinate system is defined as: the $X$-axis is from the front to the rear of vehicle, the $Y$-axis is towards the right, and the $Z$-axis is upward; The principal-axis-of-inertia coordinate system is determined by the eigenvectors corresponding to the matrix eigenvalues $\left(I_{X X}, I_{Y Y}\right.$, and $\left.I_{Z Z}\right)$.

(2) From the three principal moments of inertia $\left(I_{X X}, I_{Y Y}, I_{Z Z}\right)$ and total mass $(m)$, calculate the side lengths $(a, b, c)$ and density $(\rho)$ of the engine inertial body by following calculation formulae:

$$
a=\sqrt{\frac{6\left(-I_{X X}+I_{Y Y}+I_{Z Z}\right)}{m}}, \quad b=\sqrt{\frac{6\left(I_{X X}-I_{Y Y}+I_{Z Z}\right)}{m}}, c=\sqrt{\frac{6\left(I_{X X}+I_{Y Y}-I_{Z Z}\right)}{m}}, \rho=\frac{m}{a b c}
$$

(3) Construct an equivalent inertial body of powertrain with the centroid being at the origin of coordinates, and side lengths being $a, b, c$ respectively, and the density being $\rho$ in the principal-axis-of-inertia coordinate system $O X Y Z$ in ANSYS software, as shown in Fig.3. 
(4) Based on the direction cosine matrix $A$, calculate the three rotational angles $\left(\theta_{X}, \theta_{Y}, \theta_{Z}\right)$ formed by rotating the equivalent inertial cube of engine to the engine coordinate system along the three coordinate axes of the engine coordinate system oxyz separately .

(5) Transpose the equivalent inertial body in the principal-axis-of-inertia coordinate system to the vehicle-body coordinate system OXYZ: by placing the origin of the principal-axis-of-inertia coordinate system, i.e., the centroid of the inertial body, at the mass center of the engine in the vehicle-body coordinate system, and rotating the inertial body and the principal-axis-of-inertia coordinate system by $\theta_{Z}, \theta_{Y}$ and $\theta_{X}$ around the coordinate axes of the engine coordinate system respectively, as shown in Fig.4.

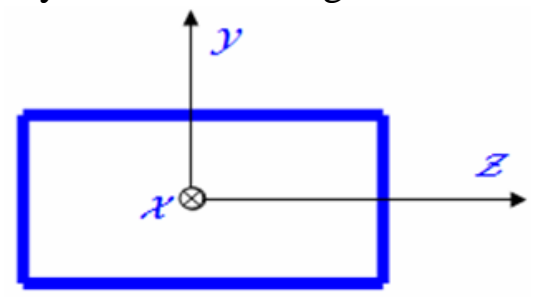

Fig.3 Inertial body in principal-axis-of-inertia coordinate system

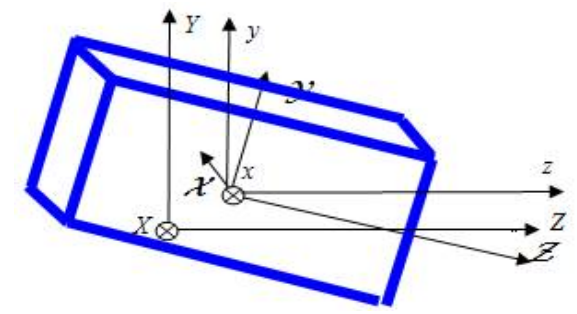

Fig.4 Inertial body in vehicle-body coordinate system

(6) Construct a simplified elastic body of engine powertrain in the vehicle-body coordinate system $O X Y Z$, and combine the equivalent inertial body and the equivalent elastic body into one by means of Boolean operation in ANSYS. Finally, the elastic body is bonded with the inertial body to result in an equivalent body of the engine powertrain, as shown in Fig. 2.

The ratio of three side lengths of the equivalent inertial body as well as their positions and angles with respect to the equivalent elastic body reflects the mass distribution law of an engine powertrain. For example, Fig. 2 shows that the mass in $Z$ direction of this engine powertrain is more than those in the other two directions, the mass is distributed from the top left at which the cylinder is to the bottom right at which the gearbox is, which agrees with the actual case. If the mass distribution does not agree with the actual case, then it indicates that the engine powertrain parameters including moments of inertia and products of inertia provided by the manufacturer are erroneous.

\section{Modal analysis in ANSYS}

For meshing the equivalent body of engine powertrain, since the engine powertrain is deemed a rigid body in modal analysis, the deformation stress of the engine powertrain can be disregarded and the meshing density can be very sparse, as shown in Fig.5.

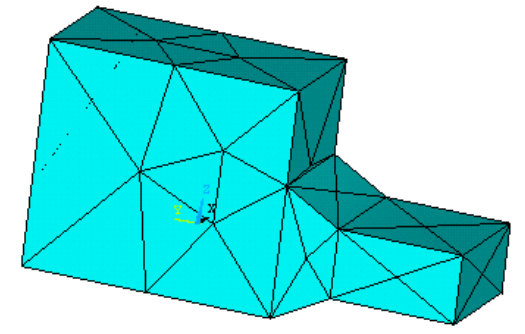

Fig.5 Mesh of equivalent analysis model

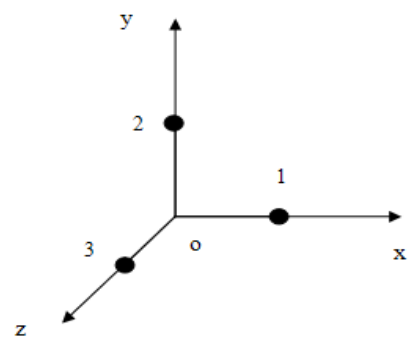

Fig.6 Mode-shape calculation model

Each mounting component is simplified as three springs perpendicular to each other at its actual accurate mounting position with the stiffness of the mounting component in corresponding direction, and one end of each spring is connected to the equivalent body while the other end is connected to the vehicle frame serving as the foundation. Finally, after setting proper parameters for the solving process in ANSYS, modal analysis of this engine powertrain mounting system can be done, then the six-order natural frequencies can be obtained. 
In modal analysis, three key points (1, 2, and 3) are set at unit length positions in three coordinate axis directions of the engine mass-center coordinate system oxyz, as shown in Fig.6. After conducting the modal analysis in ANSYS, extracting the displacements of such three points and the origin of coordinates under various orders of frequency separately, and the mode shape of this mounting system, $\{q\}=\left[\begin{array}{lllllll}x & y & z & \theta_{X} & \theta_{y} & \theta_{z}\end{array}\right]^{T}$, can be obtained with following calculation formulae:

$$
x=u_{O}, \quad y=v_{O}, \quad z=w_{O}, \quad \theta_{X}=w_{2}-w_{O}, \quad \theta_{y}=w_{O}-w_{1}, \quad \theta_{Z}=v_{1}-v_{O}
$$

where, $u_{0}, v_{0}$, and $w_{0}$ are the displacements of the engine mass center in $x, y$ and $z$ directions respectively; and $v_{1}, w_{1}$ and $w_{2}$ are the displacements of key point 1 in $y$ and $z$ directions and the displacement of key point 2 in $z$ direction respectively. Finally, the decoupling degrees can be obtained according to calculation formulae Eq. (2).

\section{Example}

The basic parameters of an automotive engine powertrain with three mounting components are shown in Tables 1 and 2; the coordinate values and the stiffness values of all mounting components in vehicle-body coordinate system are shown in Tables 3 and 4. Each principal elastic axis of every mounting component is consistent with the corresponding axis of vehicle-body coordinate system.

Table 1. Mass and mass center position

\begin{tabular}{cccc} 
Engine & $X$ & $Y$ & $Z$ \\
$109.22 \mathrm{~kg}$ & -161.06 & 36.01 & 222.05 \\
\hline
\end{tabular}

Table 3. Coordinate values of mounting components. (mm)

\begin{tabular}{cccc}
\hline & $1 \#$ & $2 \#$ & $3 \#$ \\
\hline$X$ & 77.03 & -205.32 & -152.03 \\
$Y$ & 30.62 & 430.01 & -372.83 \\
$Z$ & 114.17 & 411.59 & 295.70 \\
\hline
\end{tabular}

Table 2. Rotational inertia. $\left(\mathrm{kg} \cdot \mathrm{m}^{2}\right)$

$$
\begin{array}{lll}
\text { Ixx }=5.708 & \text { Ixy }=0.437 & \text { Ixz }=0.247 \\
\text { Iyx }=0.437 & \text { Iyy }=3.148 & \text { Iyz }=-0.887 \\
\text { Izx }=0.247 & \text { Izy }=-0.887 & \text { Izz }=4.399
\end{array}
$$

Table 4. Stiffness values of all mounting components. (N/mm)

\begin{tabular}{cccc}
\hline & $1 \#$ & $2 \#$ & $3 \#$ \\
\hline$K x$ & 170 & 75 & 130 \\
$K y$ & 75 & 140 & 90 \\
$K z$ & 120 & 155 & 140 \\
\hline
\end{tabular}

According to the above modeling method and procedures, three principal moments of inertia $\left(I_{X X}\right.$, $I_{Y Y}$, and $I_{Z Z}$ ) and the direction cosine matrix $A$ included angles between the principal-axis-of-inertia coordinate system $O X Y Z$ and the vehicle-body coordinate system OXYZ can calculated: $I_{X X}=2.607\left(\mathrm{~kg} \cdot \mathrm{m}^{2}\right), I_{Y Y}=4.859\left(\mathrm{~kg} \cdot \mathrm{m}^{2}\right), I_{Z Z}=5.790\left(\mathrm{~kg} \cdot \mathrm{m}^{2}\right), \quad A=\left[\begin{array}{ccc}-0.1597 & -0.0188 & 0.9870 \\ 0.8758 & -0.4640 & 0.1329 \\ 0.4555 & 0.8856 & 0.0906\end{array}\right]$.

According to Eq. (3), the side lengths ( $a, b$, and $c$ ) and density $(\rho)$ of the equivalent inertial body can be obtained: $a=665(\mathrm{~mm}), b=441(\mathrm{~mm}), c=303(\mathrm{~mm}), \rho=1.23 \mathrm{E}-6\left(\mathrm{~kg} / \mathrm{mm}^{3}\right)$.

From the cosine matrix $A$, the three rotational angles $\theta_{X}=-55.72^{\circ}, \theta_{Y}=80.75^{\circ}$, and $\theta_{Z}=173.3^{\circ}$ can be obtained. After establishing an equivalent model of the powertrain mounting system, performing modal analysis and extracting the displacements of various key points in ANSYS, the natural frequencies and corresponding mode shapes of the mounting system can be calculated, as shown in Table 5. And the energy decoupling degrees can be calculated with Eq. 2, shown in Table 6. The calculation result is consistent with the results with the method in Ref.[4-7].

Table 5. Mode shapes corresponding to various orders of frequencies. 


\begin{tabular}{ccccccc}
\hline Frequency $(\mathrm{Hz})$ & \multicolumn{7}{c}{ Mode shapes } \\
\hline 7.9 & $2.7 \mathrm{E}-2$ & $6.5 \mathrm{E}-2$ & $-4.5 \mathrm{E}-2$ & $6.4 \mathrm{E}-5$ & $-2.8 \mathrm{E}-4$ & $-5.6 \mathrm{E}-5$ \\
8.2 & $-7.8 \mathrm{E}-3$ & $6.6 \mathrm{E}-2$ & $4.8 \mathrm{E}-2$ & $2.6 \mathrm{E}-5$ & $2.7 \mathrm{E}-4$ & $-3.2 \mathrm{E}-5$ \\
9.1 & $8.9 \mathrm{E}-2$ & $-1.6 \mathrm{E}-2$ & $2.1 \mathrm{E}-2$ & $-1.1 \mathrm{E}-5$ & $5.5 \mathrm{E}-5$ & $-8.6 \mathrm{E}-5$ \\
11.3 & $2.6 \mathrm{E}-3$ & $-2.6 \mathrm{E}-3$ & $-6.5 \mathrm{E}-2$ & $-7.5 \mathrm{E}-6$ & $3.9 \mathrm{E}-4$ & $-6.8 \mathrm{E}-5$ \\
15.5 & $1.8 \mathrm{E}-2$ & $4.3 \mathrm{E}-3$ & $-6.2 \mathrm{E}-3$ & $1.0 \mathrm{E}-4$ & $1.8 \mathrm{E}-4$ & $4.5 \mathrm{E}-4$ \\
15.6 & $5.9 \mathrm{E}-3$ & $1.6 \mathrm{E}-2$ & $-5.0 \mathrm{E}-3$ & $-4.0 \mathrm{E}-4$ & $8.2 \mathrm{E}-5$ & $1.4 \mathrm{E}-4$ \\
\hline
\end{tabular}

Table 6. Energy decoupling degrees in various directions

\begin{tabular}{ccccccc}
\hline Direction & $X$ & $Y$ & $Z$ & $R X$ & $R Y$ & $R Z$ \\
\hline Frequency (Hz) & 9.1 & 8.2 & 11.3 & 15.6 & 11.3 & 15.5 \\
Decoupling degree (\%) & 87 & 48 & 46 & 90 & 49 & 84 \\
\hline
\end{tabular}

\section{Conclusions}

This paper proposed an equivalent analysis model method for an engine mounting system modal analysis in the ANSYS software. The engine powertrain was equivalent to a composite of one inertial body and one elastic body, the mass and the rotational inertia of real engine powertrain were accurately reflected by the composite body; every mounting component was simplified as an undamped spring along its principal elastic axes. Using this equivalent analysis model, a modal analysis and energy decoupling degree calculations of an engine powertrain mounting system example was carried out. The results showed that this equivalent modeling method is validity and feasibility for engine powertrain mounting system modal analysis.

In future, with the help of this equivalent analysis model, frequency and energy decoupling degree optimization of an engine powertrain mounting systems will be easily carried out in ANSYS, so much as dynamic response analysis and vibration isolation rate optimization of mounting systems.

\section{Acknowledgements}

This work was financially supported by the National Natural Science Foundation of China (NSFC, 50965002).

\section{References}

[1] Taeseok Jeong, Rajendra Singh: Joural of sound and vibration Vol. 234(2000), pp. 85-114

[2] Yunhe Yu, Nagi G. Naganathan, Rao V. Dukkipati: Mechanism and Machine Theory Vol. 36(2001), pp. 123-142

[3] E. Courteille, L. Léotoing, F. Mortier, E. Ragneau: European Journal of Mechanics A/Solids Vol. 24(2005), pp. 929-943

[4] Lü Zhaoping: Automotive Engineering Vol. 6(2008), pp. 523-526 (in Chinese)

[5] Chen Shuxun, Wu Song, Yin Guobao, Li Zhiqiang: Science and Technology Innovation Herald Vol. 10(2010), pp. 90-91 (in Chinese)

[6] Zhou Mi, Hou Zhichao: Automotive Technology Vol. 9(2006), pp. 13-16 (in Chinese)

[7] Xu Wenqing, Chen Jian: China Mechanical Engineering Vol. 22(2011), pp. 878-881 (in Chinese) 